Neurosurg Focus 11 (6):Introduction, 2001 Click here to return to Table of Contents

\title{
METASTATIC SPINAL TUMORS
}

\author{
Neurosurgical Focus, December 2001 \\ Topic Editor: ZiYa L. GoKaSlan, M.D. \\ Section of Spine Program, Department of Neurosurgery, The University of Texas M. D. Anderson \\ Cancer Center, Houston, Texas
}

The management of metastatic spinal disease has evolved considerably over the last decade. Recent advances in spinal instrumentation and surgical approaches have enabled spine surgeons to treat these lesions more radically and to reconstruct the spinal column more effectively. The use of spinal stabilization in conjunction with the surgical treatment of these neoplasms has resulted in significant outcome-related improvements. At present, both neurological preservation/improvement and effective management of pain can be achieved in most patients with metastatic spinal disease. The introduction of vertebroplasty/kyphoplasty has allowed patients with not only osteoporotic compression fractures but also pathological collapse to undergo a relatively noninvasive procedure for vertebral body reconstruction by which to achieve pain control. Finally, recently developed computer-guided stereotaxic radiation delivery methods (such as the CyberKnife) have enhanced our ability to administer potentially lethal doses of radiation to spinal lesions with pinpoint accuracy.

This issue of Neurosurgical Focus is dedicated to metastatic spinal tumors, and we have chosen 10 articles that address the current management of patients with these lesions and discuss some of the evolving technology. The first article, entitled "Metastatic spinal tumors," provides an excellent comprehensive review in which the authors describe the pathophysiology and management of tumors metastatic to the spine.

The second article, "Management of metastatic tumors of the spine: strategies and operative indications," is a first-rate review of decision making, available surgical approaches, and techniques used in the management of metastatic spinal tumors.
"Medical management and adjuvant therapies in spinal metastatic disease," the third article, is a well-written, thoughtful commentary. It covers the pertinent points with regard to the newer therapies such as bisphosphonates.

The next article, "Perioperative complications with costotransversectomy and anterior approaches to thoracic and thoracolumbar tumors" details two commonly used approaches for the treatment of primary and metastatic spinal tumors-namely, costotransversectomy and transcavitary routes. The authors describe the indications and patient selection criteria for each approach and demonstrate that overall complication rates were similar. The incidence of wound-related complications requiring surgery was higher in the costotransversectomy-treated group, which is a problem frequently associated with perioperative radiotherapy.

In "Image-guided spinal navigation: application to spinal metastases" the author has written a comprehensive review of frameless stereotactic spinal surgery and its application to metastatic spinal tumors. The author provides a superior review of the technology and addresses the use of this navigational method in primarily placing pedicle screws in the cervical, thoracic, and lumbar spine. The merits of this technique as an adjunct in resecting an upper thoracic neoplastic lesion are also illustrated.

In the next article, "Image-guided radiosurgery in the treatment of spinal metastases," the authors discuss the use of the CyberKnife frameless image-guided radiosurgery unit in the treatment of metastatic spinal tumors. This is a very promising technology that is likely to become the best and most accurate means of delivering radiation to tumors in and around the spinal cord. Specific issues with respect to movement of the spine and measures to correct 


\section{Z. L. Gokaslan}

for it have been addressed. The authors demonstrate that the desired dosimetry can be achieved with accuracy and that movement can be detected so that necessary adjustments in the treatment plan can be made.

"Stereotactic intensity-modulation radiation therapy for vertebral body and paraspinal tumors" is another wellwritten manuscript detailing the use of intensity-modulated radiation therapy in the treatment of metastatic spinal and paraspinal tumors. A computer-controlled multileaf collimator provides the energy with high conformality, while a noninvasive body frame minimizes intraoperative spinal movement. The authors report their limited experience in treating six patients. This method of delivering stereotactic radiation to the spine and surrounding structures has the potential for widespread use.

The authors describe a promising new stereotactic system for external use in "Ultrasonographic guidance for spinal extracranial radiosurgery: technique and application for metastatic spinal lesions." They present a case illustration in which this method was used to treat a metastatic paraspinal lesion in a patient who had previously undergone radiotherapy. This is a frameless system that relies on the matching of three-dimensional computerized tomography/magnetic resonance imaging data with optically tracked real-time ultrasound images. The system has been shown to achieve accuracy to within $1.5 \mathrm{~mm}$. Although this is a very preliminary report, the technology appears capable of being used extensively.

The authors of another paper "The role of vertebroplasty in metastatic spinal disease" address the technique of vertebroplasty and its application in patients with metastatic spinal disease. Indications and relative contraindications of the procedure are discussed in detail, and review of the current topic-related literature is also provided.

Our final article, entitled "Evaluation and treatment of spinal metastases: an overview," is a comprehensive review of the literature on the management of spinal metastases. This article is very well written, reflecting the senior author's view and experience, and it describes a sound approach to determining patient selection.

I think you will enjoy reading all of the articles published here on our topic of Metastatic Spinal Tumors. Thank you.

Z. L. Gokaslan, M.D. 